\title{
Optimising gravitational waves follow-up using galaxies stellar mass
}

\author{
J. -G. Ducoin ${ }^{1}$, D. Corre $^{1}$, N. Leroy ${ }^{1}$, E. Le Floch $^{2}$ \\ ${ }^{1}$ LAL, Univ Paris-Sud, CNRS/IN2P3, Orsay, France \\ ${ }^{2} I R F U, C E A$, Univ Paris-Saclay, Gif-sur-Yvette, France
}

Accepted 2020 January 10. Received 2020 January 9; in original form 2019 November 11

\begin{abstract}
We present a new strategy to optimise the electromagnetic follow-up of gravitational wave triggers. This method is based on the widely used galaxy targeting approach where we add the stellar mass of galaxies in order to prioritise the more massive galaxies. We crossmatched the GLADE galaxy catalog with the AllWISE catalog up to 400Mpc with an efficiency of $~ 93 \%$, and derived stellar masses using a stellar-to-mass ratio using the WISE1 band luminosity. We developed a new grade to rank galaxies combining their 3D localisation probability associated to the gravitational wave event with the new stellar mass information. The efficiency of this new approach is illustrated with the GW170817 event, which shows that its host galaxy, NGC4993, is ranked at the first place using this new method. The catalog, named Mangrove, is publicly available and the ranking of galaxies is automatically provided through a dedicated web site for each gravitational wave event.
\end{abstract}

Key words: gravitational waves - catalogues - methods: observational

\section{INTRODUCTION}

Gravitational waves from binary neutron star (BNS) coalescence, in association to short gamma-ray burst, opened a new era of multi-messenger astronomy. The event of the 17th august 2017 was a real breakthrough for the multi-messenger astronomy. For the first time, an electromagnetic counterpart of a gravitational wave was observed (Abbott et al. 2017a). The association with a gamma ray burst (GRB) detected by Fermi-GBM a few seconds after the coalescence provides the first evidence of a link between BNS merger and short gamma ray burst. The huge effort of ground telescopes follow-up in association to the relative small volume of this event localisation in the sky, allowed the identification of the GW electromagnetic counterpart. The multi-wavelength observations improved our understanding of the physics of strong-gravity and put some constraints on astrophysical models related to matter during the merger and post-merger phase. A first constraint of the speed of gravitational waves and violation of Lorentz invariance has been determined from this event (Abbott et al. 2017c). Both kilonova and afterglow observations provide information about the neutron star equation of state, energy of the ejecta, merger remnant, ambient medium and so on (Metzger 2019; Abbott et al. 2018c; Hajela et al. 2019; Gill et al. 2019). Such event also provide a new independent derivation of the Hubble Constant (Abbott et al. 2017b; Coughlin et al. 2019a; Hotokezaka et al. 2019).

The third LIGO-Virgo run (O3) started the first of April 2019 and multi-messenger astronomy related to gravitational waves with it. With improved sensitivity of the LIGO-Virgo detectors, the year-long third observing run (O3) has already brought his share of merging binaries and promises many more of them. Therefore an intensive multi-wavelength follow-up of those events with ground and space instruments is performed all around the world. But the identification of the electromagnetic counterpart of such event is very challenging knowing the wide sky localization area provided by LIGO-Virgo (which can span more than $1000 \mathrm{deg}^{2}$ ) and require complex observation strategies.

Many efforts were done recently to optimise the observations for these large sky areas (Ghosh et al. 2016a; Coughlin et al. 2019b). For large field of view (FoV) telescope the standard approach consists in observing the localisation error box provided by LIGO-Virgo (Singer \& Price 2016; Veitch et al. 2015) using an optimised tiling of the sky (Coughlin et al. 2018). In such standard strategy, the scheduling of the tile observation is provided by the $2 \mathrm{D}$ probability distribution from LIGO-Virgo skymaps.

As suggested by (Gehrels et al. 2016) more recent works tried to include galaxies population to the strategy (Arcavi 
et al. 2017; Antolini et al. 2017; Rana \& Mooley 2019). Such developments allow to use the $3 \mathrm{D}$ probability distribution from the LIGO-Virgo skymap (and not only the 2D probability) to produce a "galaxy weighting" rank for the tiles. It also allows to provide a scheduling of observation for narrow FoV telescopes. Indeed, with such information we can provide a list of interesting galaxies (i.e. ranked by their $3 \mathrm{D}$ position probability inside the LIGO-Virgo skymap) to be observed by these small FoV telescopes. To allow the use of information related to galaxies, one must rely on a galaxy catalog that is sufficiently complete compared to the interferometers sensitivity range. The current binary neutron star range is at $130 \mathrm{Mpc}$ for LIGO Livingston, 110 Mpc for LIGO Hanford, and $50 \mathrm{Mpc}$ for Virgo (Abbott et al. 2018a). The CLU catalog (Cook et al. 2019) for the north hemisphere contains the WISE1 luminosity information as well as spectroscopic measurement of local galaxies, however it is non publicly available. Therefore, for the purpose of our work, we rely on the publicly available GLADE galaxy catalog (Dálya et al. 2018) which is all-sky and complete up to $100 \mathrm{Mpc}$, and nearly complete up to $150 \mathrm{Mpc}$.

Given the large size of error boxes, the number of galaxies compatible with an event can be very large ( $>$ few thousands). In such cases, the classification using the 3D probability only is limited because it will produce similar values for a large number of galaxies. Adding galaxy properties to the ranking is a way to reduce the sample size of interesting galaxies. Among the various galaxies properties that could influence the rate of BNS merger, such as star formation rate (SFR), stellar mass and metallicity, several works pointed out a significant dependence to the stellar mass (Artale et al. 2019; Toffano et al. 2019; Mapelli et al. 2018). Furthermore, short GRB host galaxies are known to be associated to BNS merger since GW170817, and are found in massive galaxies. The short GRB host galaxies are more massive than the long GRBs host galaxies, pointing to the importance of the stellar mass in determining the rate of short GRBs (Leibler \& Berger 2010; Fong et al. 2013; Berger 2014). So far, the only one known (by gravitational waves) host galaxy of BNS merger is NGC4993 from GW170817 event. This galaxy present a very high mass and a low star formation rates (Im et al. 2017; Blanchard et al. 2017; Hjorth et al. 2017; Levan et al. 2017; Pan et al. 2017). The host galaxy of GRB150101B presented as a analogue of GRB170817A (Troja et al. 2018) is also a vary massive galaxy. In the light of those information we chose to focus on the stellar mass for the selection of gravitational waves host galaxy candidates.

In Section 2, we describe the general galaxy targeting approach and the new formulation we propose to include the stellar mass information. In the Section 3 we describe the crossmatch between the GLADE and AllWISE catalogs and the stellar mass estimation as well as an estimation of the completeness of the resulting Mangrove (Mass AssociatioN for GRavitational waves ObserVations Efficiency) catalog in terms of stellar mass. In Section 4 we test our method on the GW170817 event. In Section 5 we discuss future development of this method for wide field of view telescopes. Throughout this paper, we use the Plank 2015 cosmological parameters (Planck Collaboration 2016).

\section{GALAXY TARGETING METHOD}

In this section, we first describe the standard galaxy approach using the 3D localisation of the GW skymap, and then we propose a new formulation of the grade used to rank galaxies in order to include the stellar mass information.

\subsection{Standard approach}

In case of a gravitational wave event, LIGO-Virgo rapidly releases a probability skymap based on the distance and two dimensional localisation of the event allowing to constrain the region of the sky to search for the GW electromagnetic counterpart (Singer \& Price 2016). With such skymap we are able to fetch the probability density per unit of volume at a given position (Singer et al. 2016). This is used to infer the probability of a given galaxy to be the host of the merger according to its celestial position $P_{\text {pos }}$ with the following relation:

$P_{\text {pos }}=P_{d V}=\frac{P_{\text {pixel }}}{\text { Pixel area }} N_{\text {pixel }} e^{-\frac{1}{2}\left(\frac{D_{\text {galaxy }}-\mu_{\text {pixel }}}{\sigma_{\text {pixel }}}\right)^{2}}$

Where $P_{\text {pixel }}$ is the $2 \mathrm{D}$ probability included in the given pixel, $N_{\text {pixel }}$ is the normalisation factor for the given pixel, $\mu_{\text {pixel }}$ is the mean distance value at the given pixel, $\sigma_{\text {pixel }}$ is the standard deviation at the given pixel and $D_{\text {galaxy }}$ is the luminosity distance of the galaxy fetched from the galaxy catalog. The outputs of the LIGO-Virgo localization pipelines are HEALPix (Hierarchical Equal Area isoLatitude Pixelization) all-sky images, the skymap we are dealing with is composed of pixels defined by the HEALPix (Górski et al. 2005) format.

For the selection of the galaxies, we classified as "compatible" with the skymap, a galaxy which fulfills the two following conditions:

- Its $2 \mathrm{D}$ position in the sky as to be in the $90 \%$ of the $2 \mathrm{D}$ skymap probability distribution.

- Its distance has to fall within the 3 sigma distance error localization at the given pixel of the galaxy.

With such conditions we ensure that telescopes will not point outside of the $90 \%$ skymap probability distribution. The conservative choice of $3 \sigma$ on the distance constraint is motivated by the fact that galaxies with a low distance probability will be always penalised in the ranking process. Regarding the condition on the distance, we use the pixel by pixel information and not the mean distance estimation for the LIGO-Virgo candidate, as in the approach adopted by (Arcavi et al. 2017). This is more efficient because the distance estimation can be very inhomogeneous in a given skymap. The figure 1 shows the distribution pixel by pixel of the mean distance $\mu$ and the standard deviation $\sigma$ inside the the $90 \%$ of the 2D skymap probability distribution for the S190425z candidate during the O3 run. This example of a BNS candidate shows that for a large portion of the skymap the $\mu$ and $\sigma$ at a given pixel are far away from the

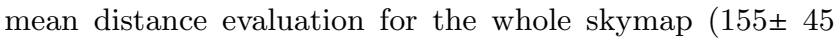
$\mathrm{Mpc}$ in this case). Using pixel by pixel information prevents to dismiss compatible galaxies or select incompatible ones. One of the main advantage of the galaxy targeting is to reduce the amount of observations necessary to cover a 


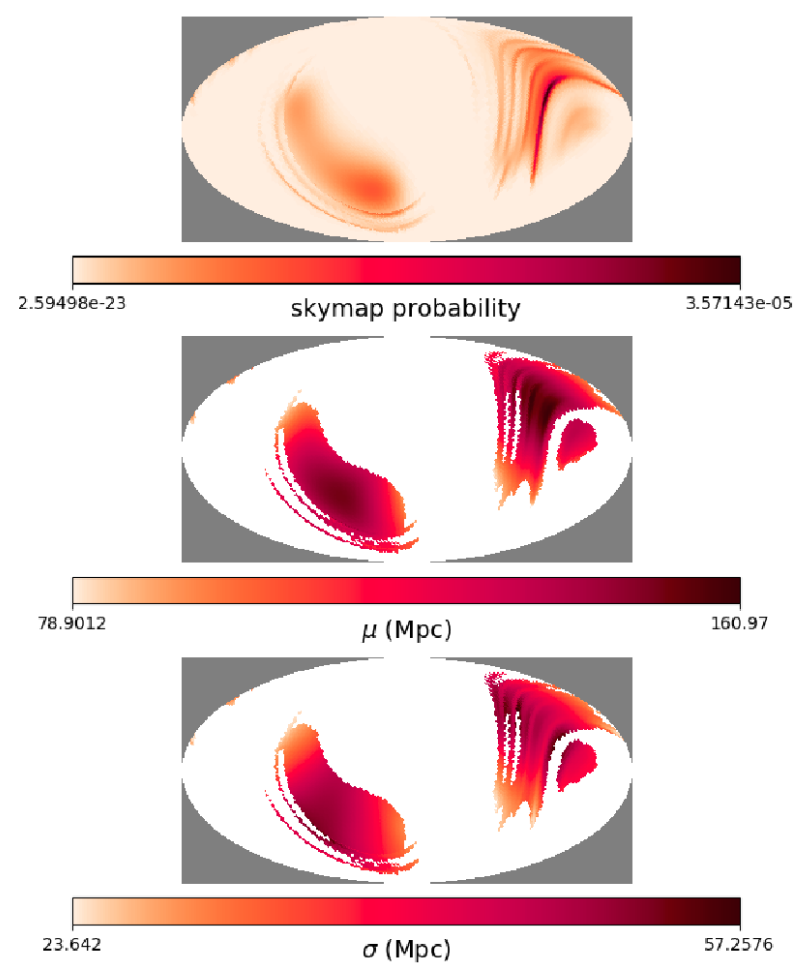

Figure 1. The top plot show the probability distribution for the BNS candidate S190425z of O3. The middle and bottom plot are respectively the mean distance and its standard deviation distributions for the pixels inside the the $90 \%$ of the 2D skymap probability distribution

given skymap. The figure 2 shows the result of the galaxy targeting for GW170817 with a standard field of view of 20 arcmin. A large part of the skymap is not worth observing because it does not contain any compatible galaxies. A total of 44 pointing are needed to observe all galaxies compatible with the $90 \%$ skymap whereas it would have required 144 tiles to cover the entire $90 \%$ skymap, which results on significant gain of observational time allocation and revisits.

In such a standard strategy only the $3 \mathrm{D}$ position of the galaxy is used, and a more advanced strategy is to include physical properties of the galaxies such as the stellar mass as the host galaxy of BNS counterparts are likely to be found in massive galaxies.

\subsection{Grade reformulation with stellar mass}

In this section, we describe how to take into account the stellar mass information in the galaxy ranking process, in order to prioritize massive galaxies. We introduce a new term $G_{\text {mass }}$ defined as:

$G_{\text {mass }}=\frac{M_{*, \text { galaxy }}}{\sum M_{*, \text { galaxy }}}$

where $M_{*, \text { galaxy }}$ is the stellar mass of a given galaxy and the sum is over all of the galaxies compatibles with a given skymap (see 2 for the definition). This We first combine this term to the standard grade defined in equation (1) for each

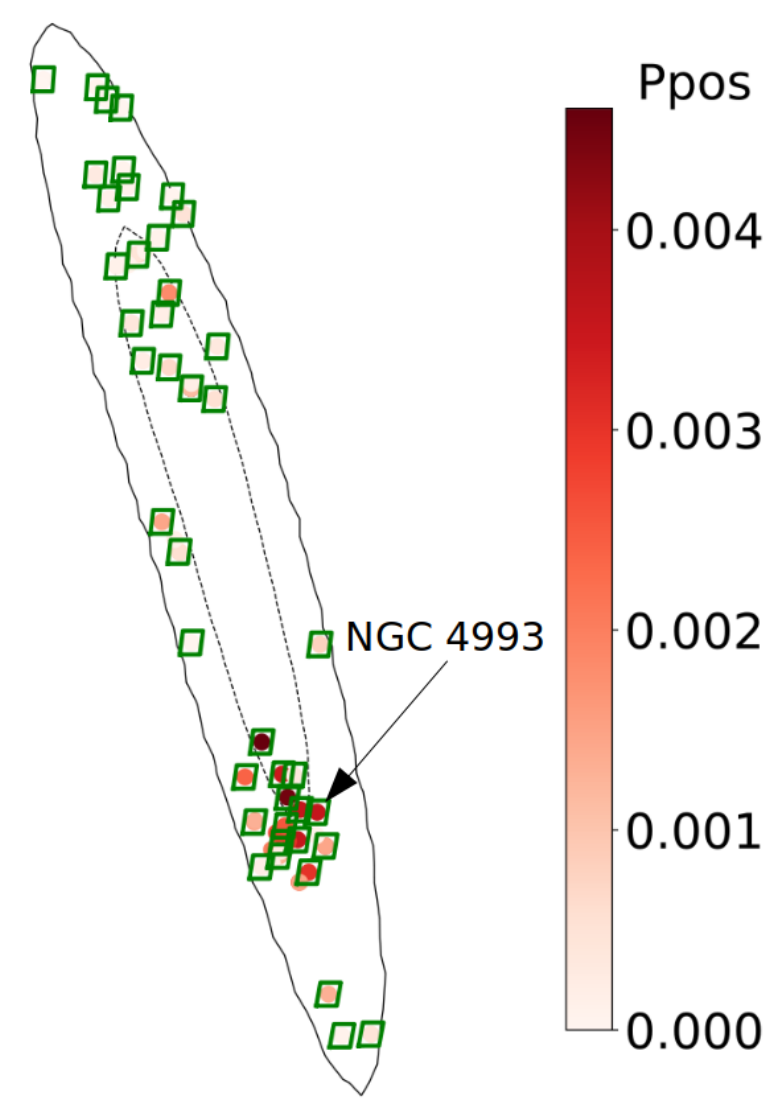

Figure 2. Skymap of GW170817. The dashed and the solid line enclose respectively the $50 \%$ and the $90 \%$ of the skymap. The green squares represent the pointing of a telescope with a field of view of 20'. The red color scale show the grade of the galaxies according to (3). The black arrow show the position of the GW host galaxy, NGC 4993.

galaxy computing the product as:

$G_{\text {tot }}=P_{\text {pos }} \times G_{\text {mass }}$

The equation (3) is proposed to fit with (Arcavi et al. 2017) expression to allow a direct comparison of the results (see Section 4), but the drawback of this expression with a simple product is that galaxies for which no stellar mass is available are simply not considered. In order to keep galaxies without stellar mass information, and still use their 3D localisation probability, $P_{\text {pos }}$ from equation (1), we propose to redefine the grade defined in equation (3) as:

$G_{\text {tot }}=P_{\text {pos }}\left(1+\alpha \beta G_{\text {mass }}\right)$

where $\alpha$ and $\beta$ are positive real parameters. With such definition, $G_{\text {mass }}$ is set to 0 when the stellar mass information is not available to fall back on $P_{p o s}$. The parameter $\alpha$ is defined such that the two terms in equation (4) contribute equally to the total grade, $G_{\text {tot }}$ :

$\frac{\sum P_{\text {pos }}}{N}=\frac{\sum P_{\text {pos }} \alpha G_{\text {mass }}}{N}$

$\Rightarrow \alpha=\frac{\sum P_{\text {pos }}}{\sum P_{\text {pos }} G_{\text {mass }}}$ 
where $\mathrm{N}$ is the total number of galaxies compatible for a given skymap having a determined stellar mass, and the sum is also over all galaxies compatible for a given skymap having a determined stellar mass. The parameter $\beta$ is used to weight the importance of $G_{\text {mass }}$ in the total grade, it is skymap independent. Ideally, $\beta$ should be fitted on a statistically significant sample of gravitational wave host galaxies, but as only one event has been detected so far, we simply chose to put $\beta$ equal to one.

Previous works (Arcavi et al. (2017); Salafia et al. (2017)) chose to include an other factor of the grade which describe the likelihood to detect the counterpart according to limiting magnitude of the observing telescope and the expected magnitude of the source. Such factor can be added to the expression (3) and (4) if needed. We choose not to develop such strategy because first the limiting magnitude of a telescope can vary a lot between two observations (seeing, horizon...) and secondly only one detection of gravitational wave electromagnetic counterpart has been achieved at the moment, so only one one set of data describing the expected kilonova lightcurve is available and assuming a standard lightcurve on a single object could be risky.

The reformulation of the grade presented here is independent of the method used to determine the stellar mass of galaxies. In section 3 we present the method we have chosen to follow to obtain the stellar mass of galaxies in a homogeneous way for a catalog over the whole sky. The resulting catalog is fully publicly available as described in section 5

\section{ADDING STELLAR MASS TO GLADE CATALOG}

In this section we aim at adding the stellar mass for the GLADE galaxy catalog. In the first part, we describe how to derive the stellar mass from the WISE1 band luminosity. In the second part we study the completeness of the Mangrove catalog resulting from the crossmatch between GLADE and AllWISE catalogs.

\subsection{WISE1 band as a prob of stellar mass}

Homogeneity in the stellar mass estimation is crucial for our purpose as it is used to rank the galaxies. Unfortunately, the GLADE galaxy catalog we rely on provides the B, J, $\mathrm{H}$ and $\mathrm{K}$ band magnitudes for some of the galaxies but not the stellar mass. We did not chose to compile the stellar masses from various works for homogeneity reasons as it would bring systematics in the ranking process due to possibly quite different methods in the stellar mass estimation. Previous work (Arcavi et al. 2017) used the B band magnitude as an indicator of the stellar mass. However, the B band is sensitive to the star formation history and can be strongly affected by dust extinction (Cardelli et al. 1989; Fitzpatrick \& Massa 2007). The near-infrared luminosity emitted by the old stellar population is fairly insensitive to dust extinction, and is thus considered as a reliable indicator of the total stellar mass of a galaxy (Bruzual \& Charlot 2003; Maraston 2005).

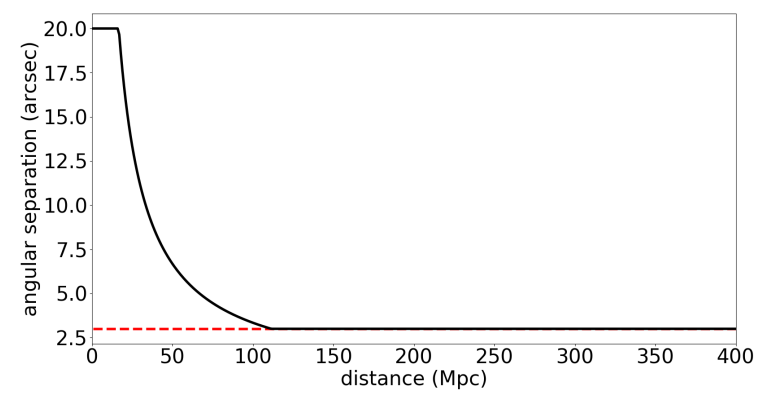

Figure 3. Angular separation condition used for the crossmatch as a function of the distance. The red dashed line show the limit value of 3 arcseconds.

In our work, we restrict the distance to $400 \mathrm{Mpc}$ as it is reasonably above the limiting sensibility distance of LIGOVirgo for binary neutron star merger with O3 sensibility (Chen et al. 2017). Up to $400 \mathrm{Mpc}$, there are only $\sim 67 \%$ of the galaxies with a $\mathrm{K}$ band magnitude in the version 2.3 of the GLADE catalog. As showed by (Kettlety et al. 2018; Norris et al. 2014), the WISE1 $(3.4 \mu \mathrm{m})$ luminosity is a reliable indicator of the stellar mass, moreover the AllWISE catalog (Cutri \& et al. 2014) has the advantage to be an all-sky catalog. Therefore we made the choice to perform a crossmatch between the GLADE and the AllWISE catalogs to derive a reliable stellar mass estimation.

From WISE1 luminosities, (Kettlety et al. 2018; Norris et al. 2014) showed that the stellar mass of galaxies can be reliably estimated with a constant mass to light ratio: $\Upsilon_{*}^{3.4 \mu m} \sim 0.60 M_{\odot} / L_{\odot, 3.4 \mu m}$ where $M_{\odot} / L_{\odot, 3.4 \mu m}$ is the mass-to-light ratio in units of solar masses over the solar luminosity in the WISE $3.4 \mu \mathrm{m}$ band $\left(m_{\odot}, 3.4 \mu m=3.24 m a g ; L_{\odot}, 3.4 \mu m=1.58 \times 10^{32} \mathrm{ergs}^{-1}\right.$; (Jarrett et al. 2013)). This approach derives stellar mass with an error of 0.10 dex (Kettlety et al. 2018; Norris et al. 2014). Although the value of $\Upsilon_{*}^{3.4 \mu m}$ can vary from $\sim 0.5$ to $\sim 0.65$ in the literature, this results on small changes on the derived stellar mass and will not affect the ranking of the galaxies as it is a constant ratio.

We spatially crossmatched the GLADE catalog cut to $400 \mathrm{Mpc}$ with the AllWISE catalog using a radius crossmatch varying with the distance as seen in Figure 3. The radius is defined as 5\% the angular diameter of the milky way $(0.0324 \mathrm{Mpc})$ and we impose a minimum and maximum of 3 and 20 arcseconds respectively. This strategy was chosen to optimize the match at low distance where galaxies can have very large angular size. A same AllWISE object can appear more than once in the preliminary crossmatched catalog, meaning that there is more than one GLADE galaxy around the given AllWISE object. For such case, knowing the angular resolution of the WISE telescope in the WISE1 band (6.1 arc seconds), we only kept the closest object if it is the only one in a radius of 6.1 arc seconds around the GLADE galaxy. The presence of an AGN implies a significant emission in the infrared and near-infrared (Ruiz et al. 2013; Burtscher et al. 2015; Sanders 1999), and thus biased our stellar mass estimation for such galaxies. We identify active galactic nucleus (AGN) 
from the resulting catalog using the mid-infrared color criterion $W 1-W 2 \geq 0.8$ as used in (Stern et al. 2012; Assef et al. 2018). This corresponds to 3346 galaxies, that are still present in the catalog but without stellar mass estimation. We use the elliptical aperture photometry flux from ALLWISE catalog whenever available for the source, otherwise the profile fitting photometry is used. This ensures that these fluxes should encompass each galaxy full radial extent. At the end we obtain the stellar mass information for 743780 objects, knowing that GLADE catalog cut to $400 \mathrm{Mpc}$ have 800986 galaxies we have a $\sim 93 \%$ of match efficiency.

Before the conversion of WISE1 magnitudes to stellar masses using the mass-to-light ratio provided by (Kettlety et al. 2018), we apply a K-correction to correct for the distance. We use 31 SED templates covering all known type of galaxies, from red elliptical to blue star-forming galaxies (Ilbert et al. 2009). We compute the K-correction both without dust attenuation and with $E(B-V)=0.5$ mag using the Calzetti attenuation law (Calzetti et al. 2000). The $\mathrm{K}$-correction is insensitive to the galaxy type and dust attenuation up to $\mathrm{z}=0.12$ as seen in Figure 4. Given the distance limitation of $400 \mathrm{Mpc}$ ( $\mathrm{z} 0.085$ ) for our catalog, we computed the $\mathrm{K}$-correction at a given distance as the mean value for the 31 galaxies SED templates with and and without dust attenuation. At this range of distances, the effect is negligible, however this will become important for future catalogs that will need to be deeper to encompass the sensitivity improvement of LIGO-Virgo interferometers. We recall that all stellar masses used in this work were derived within this work using the same method to ensure homogeneity. Regarding the reliability of our stellar mass estimates, although we aimed at an homogeneous estimation, (Kettlety et al. 2018) showed that for low redshift galaxies the stellar mass estimation using a constant mas-to-light ration using the WISE1 luminosity is in good agreement with the one predicted from a more elaborate SED fitting technique.

\subsection{Completeness of the Mangrove catalog}

In this section, we aim at quantifying the completeness of the Mangrove catalog in terms of stellar mass. The stellar mass function is well described by a Schechter function (Schechter 1976), $\Phi(M)$, which parametrises the number density of galaxies, $n_{\text {galaxies }}$, as a function of their stellar mass. In this work, we use the low-redshift galaxy stellar mass function derived from the Galaxy And Mass Assembly (Wright et al. 2017), using a double Schechter function in logarithmic mass space defined as:

$$
\begin{aligned}
n_{\text {galaxies }} & =\Phi(M) d \log M \\
& =\ln (10) e^{-10^{\log M-\log M^{*}}}\left[\Phi_{1}^{*} \cdot\left(10^{\log M-\log M^{*}}\right)^{\alpha_{1}+1}\right. \\
& \left.+\Phi_{2}^{*} \cdot\left(10^{\log M-\log M^{*}}\right)^{\alpha_{2}+1}\right] d \log M
\end{aligned}
$$

where $\log M^{*}=10.78, \Phi_{1}^{*}=2.93 \cdot 10^{-3} h^{3} \cdot M p c^{-3}, \Phi_{2}^{*}=$ $0.63 \cdot 10^{-3} h^{3} \cdot M p c^{-3}, \alpha_{1}=-0.62$ and $\alpha_{2}=-1.5$ (Wright et al. 2017).



Figure 4. K-correction for the 31 galaxy SED templates for the redshift range 0 to 2 with a zoom-in from 0 to 0.2 . The blue solid lines are for the SED templates without dust attenuation, red solid lines are with a dust attenuation of $E(B-V)=0.5 \mathrm{mag}$ using the Calzetti law (Calzetti et al. 2000). The green dashed line is the mean value at a given distance of the K-correction for all galaxies SED considered in this study. The black dashed line corresponds to $400 \mathrm{Mpc}$.

Following the approach of (Gehrels et al. 2016; Dálya et al. 2018) regarding the luminosity function, we divided galaxies into 12 luminosity distance shells, with a width of $33.3 \mathrm{Mpc}$. For each shell, we construct histograms of WISE1 band derived stellar mass and integrate the double Schechter function for the same stellar mass bins in Figure 6. As the luminosity distance increases more and more low mass galaxies are missing. Regarding the double Schechter function derived from GAMA, the new catalog is fairly complete up to $33 \mathrm{Mpc}$ in terms of stellar mass. In this work, we are interested in the more massive galaxies, so we computed the stellar mass, $M_{1 / 2}$, for which half of the stellar mass density is contributed by galaxies below and above this value. By computing $\int_{\log M_{1 / 2}}^{13} \log M \Phi(M) d \log M=0.5 *$ $\int_{7}^{13} \log M \Phi(M) d \log M$, we find $\log M_{1 / 2}=10.674$. For all the luminosity distance shells, the Mangrove catalog distribution follows the double Schechter function for $\log M>\log M_{1 / 2}$ as seen in Figure 6. Our method is prioritising the more massive galaxies, consequently the fairly completeness relative to the double Schechter function for galaxies at $\log M$ $>\log M_{1 / 2}$ minimises the lack of low mass galaxies as the distance increases.

\section{VALIDATION ON GW170817 EVENT}

At the time the event of the 17th august 2017 is the only gravitational wave event with a detected electromagnetic counterpart so our new method has to be tested on this event.

The GW170817 released distance is $40 \pm 8 \mathrm{Mpc}$, the $90 \%$ skymap spans around $30 \mathrm{deg}^{2}$ and as shown in the figure 2 according to our criteria (see Section 2 for the definition) there is 65 compatible galaxies within. The table in Appendix A 


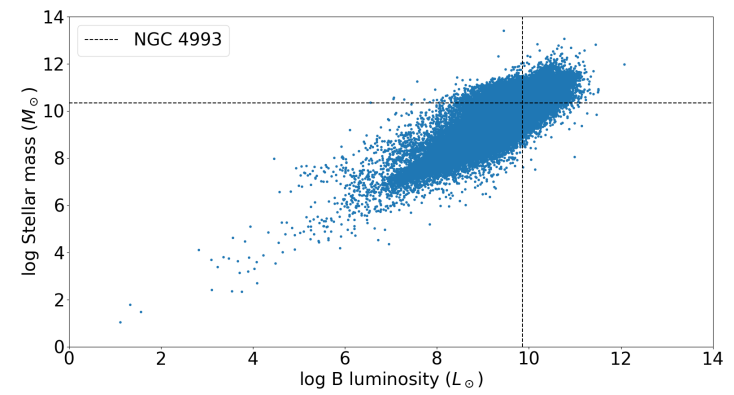

Figure 5. B band luminosity provided by GLADE as a function of the stellar mass determined with the constant mass to light ratio using the $\mathrm{W} 1$ band. The crossing of the dashed lines shows the NGC 4993 (host of GW170817) position on this plot.

shows the results for this event on the selection of galaxies and their ranking according to the standard approach (Equation 1), according to Arcavi et al. (2017), according to our method with product (Equation 3) and according to our method with addition (Equation 4). NGC 4993 host galaxy of GW170817 is ranked in $5^{\text {th }}$ position in the standard approach. The (Arcavi et al. 2017) grade improved its rank to the $2^{\text {nd }}$ position and is ranked first with our method for both (Equation 3) and (Equation 4) expressions. These results show that if we had used our grade to monitor this event, there would have been a gain in the speed of observation of the host galaxy. An important thing to see in those results is that the new grades are behaving like expected i.e. galaxies with high stellar mass are prioritized compared to galaxies with small stellar mass (see Appendix A where the list of galaxies for the four methods are reported with their grade and stellar mass if used). We also stress that galaxies with a high $3 \mathrm{D}$ probability but without stellar mass information also appear in the list (see Appendix A), for instance galaxy WINGSJ125701.38-172325.2 is ranked in $9^{\text {th }}$ position, keeping such host candidate is a real improvement of the equation 4 compared to equation 3 .

GW170817 was quite a lucky event in terms of distance of the source and good localisation thanks to data available for the three GW detectors. However, as the three inteferometers are not always in operating mode, this is very common to have a two inteferometers detection resulting in a poorer localisation (Abbott et al. 2018b). In order to test our method on a larger skymap, i.e. a two inteferometers event, we choose to use the GW170817 skymap without Virgo data. The $90 \%$ skymap spans $\sim 190 \mathrm{deg}^{2}$, this is a good example of two GW detectors localisation for which we can be sure of the counterpart host. According to our criteria in Section 2, there are 205 galaxies compatible with this skymap. The table in appendix B shows the resulting ranking for this skymap using the four presented grades. NGC 4993 host galaxy of the event is ranked in $27^{\text {th }}$ position in the standard approach. The (Arcavi et al. 2017) grade ranks it at the $6^{\text {th }}$ position and our grade is even more successful by putting NGC 4993 in the $4^{\text {th }}$ position with both equations 3 and 4 expressions. Those results shows that the gain of our method for the follow up of gravitational waves event is even bigger in the case of wide skymap. This example of wider skymap allows us to see that sometime the (Arcavi et al. 2017) grade and our grades behave differently and even oppositely. For example the galaxy PGC043966 is ranked in $37^{\text {th }}$ position with the standard grade and, when the Arcavi et al. (2017) grade upgrade its rank to the $30^{\text {th }}$, both of our grades (equations 3) and (4) downgrade its rank to the $54^{t h}$ and $49^{t h}$ position respectively. We can also note that the (Arcavi et al. 2017) grade ranked the NGC 4658 galaxy in first position due to is high $\mathrm{B}$ band luminosity but this galaxy is ranked in position 9 with our final grade definition. For these two examples the stellar mass estimation is not as high as the B band luminosity might suggest. Using the B band luminosity in those cases would have led to observe preferentially less massive galaxies. We illustrate the difference in the behavior between our grade and the one from (Arcavi et al. 2017) in Figure 5, where we plot the stellar mass estimation using WISE1 band as a function of the B band luminosity for the same galaxies. We see that for a given $\mathrm{B}$ band luminosity there is an important scatter in stellar mass spanning a few order of magnitudes. It means that the probability associated to a galaxy with respect to its stellar mass estimation can behave very differently for both methods. For example, for the host galaxy associated to GW170817, NGC 4993, we derived a stellar mass of $\sim 2.14 \times 10^{10} M_{\odot}$ but galaxies with similar B band luminosity $\left(\sim 10^{10} L_{\odot}\right)$ in the catalog span a stellar mass range from $\sim 3.8 \times 10^{7} M_{\odot}$ to $\sim 1.0 \times 10^{12} M_{\odot}$ which represents almost five order of magnitudes. In addition to this different behavior, we also illustrate the flexibility of the grade defined in equation 4 by noting that using the grade defined in equation 3 would have led not to use $\sim 7 \%$ of the galaxies inside the $400 \mathrm{Mpc}$ of Mangrove catalog, and $\sim 5 \%$ of the galaxies when using the $\mathrm{B}$ band luminosity as in (Arcavi et al. 2017). 

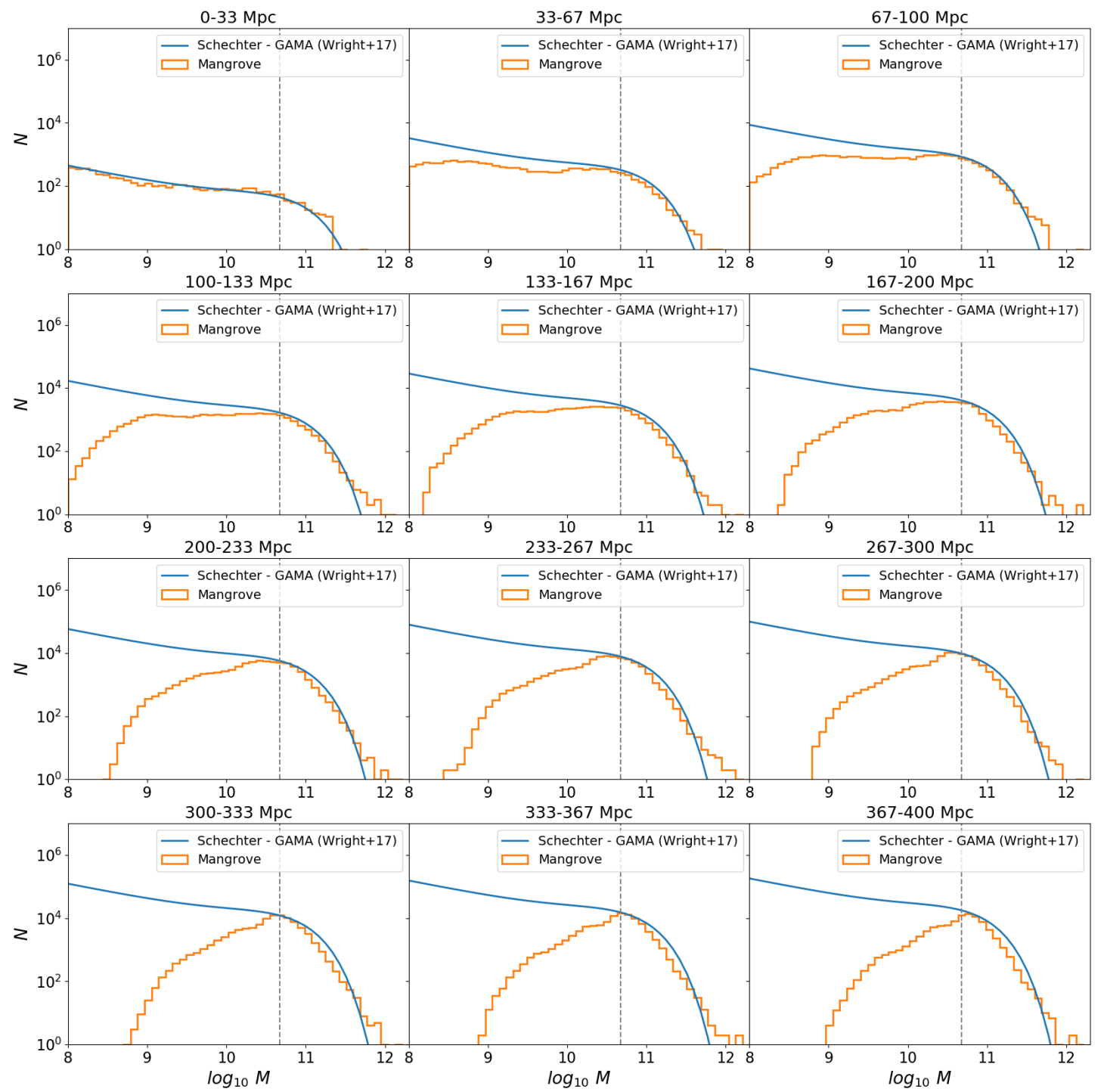

Figure 6. Stellar mass histograms for the Mangrove catalog at different luminosity distance shells compared to the double Schechter function derived from GAMA (Wright et al. 2017) weighted by the volume of each shell. Each panel is divided in 50 bins of $\log M$. The black dashed line represents the stellar mass, $\log M_{1 / 2}$ for which half of the stellar mass density is contributed by galaxies at $\log M>$ $\log M_{1 / 2}$. 


\section{DISCUSSION AND CONCLUSION}

\subsection{Utilisation of the galaxies for the tiling}

In this section we present a future development to use the galaxies for wide field of view telescopes. For the optimisation of wide FoV telescopes ( $\gtrsim 1 \mathrm{deg}^{2}$ ) follow up observations the standard approach consists in defining tiles over the sky and ranking them according to a given grade (Coughlin et al. 2018; Ghosh et al. 2016b). A first version of the grade of a tile you can build is:

$$
\text { Grade }_{\text {tile }}=\sum_{\text {pixel } \in \text { tile }} P_{2 D, \text { pixel }}
$$

where we sum up the $2 \mathrm{D}$ probability of the pixels $P_{2 D \text {,pixel }}$ within the tile. When using a catalog of galaxies one can define a "galaxy weighted" grade for the tile using the grade of the galaxies:

$$
\text { Grade }_{\text {tile }}=\sum_{\text {gal } \in \text { tile }} \text { Grade }_{\text {gal }}
$$

where we sum up the grade of the galaxies within the tile. In this expression any galaxy grade can be used, such as the expression in equation 4 that will optimise the chance to find the GW electromagnetic counterpart. The biggest issue of this approach is the catalog completeness, which makes this approach valid only below a distance threshold above which one has to switch back from grade definition of equation 9 to equation 8 in order to prevent using only galaxy information at a distance where your catalog is not complete enough. We present a reformulation of the tile grade using our developments which allows to use galaxies catalog and a galaxy weighted grade at any distances. From Section 3.2, we are able to define the mass completeness of the catalog, $C_{m 1, m 2}^{d 1, d 2}$, between distances $d_{1}$ and $d_{2}$ for a stellar mass range between $m_{1}$ and $m_{2}$ comparing to the double Schechter function:

$$
\begin{aligned}
C_{m 1, m 2}^{d 1, d 2}= & \int_{m 1}^{m 2} M_{*} f_{\text {Schecheter }}\left(M_{*}\right) d M_{*} \\
& -\int_{m 1}^{m 2} M_{*} \text { histogram }\left(M_{*}\right) d M_{*}
\end{aligned}
$$

With this parameter defining the completeness, we can define the grade of a given tile by:

$$
P_{\text {tile }}=\sum_{\text {pixel } \in \text { tile }}\left[\sum_{\text {gal } \in \text { pixel }} C_{m 1, m 2}^{d 1, d 2} P_{\text {tot }, \text { gal }}+\left(1-C_{m 1, m 2}^{d 1, d 2}\right) P_{2 D, p i x e l}\right]
$$

where the first sum over all pixels inside the tile, the second sum over all galaxies falling in the $2 \mathrm{D}$ location of the pixel, $P_{\text {tot, gal }}$ is the grade of a given galaxy defined in Section $2.2, P_{2 D \text {,pixel }}$ is the $2 \mathrm{D}$ probability of the pixel, $d_{1}$ and $d_{2}$ are chosen as $\mu_{\text {pixel }} \mp \sigma_{\text {pixel }}$ respectively and $m_{1}$ and $m_{2}$ are fixed to $10^{7}$ and $10^{13}$ (stellar mass range validity of the Schechter function fitted by Wright et al. (2017)). This expression removes any distance limitation for the use of galaxies weighted tiles. Note that with this expression we simply sump up the probability of the galaxies within a tile when the catalog is complete, whereas we sum up the $2 \mathrm{D}$ probability of all pixels within a tile when the catalog is not complete.

\subsection{Conclusion}

The electromagnetic follow-up of gravitational wave events is very challenging, the poor localisation of the source provided by LIGO-Virgo forces telescopes around the world to observe large areas of the sky. As the electromagnetic counterpart is expected to decay rapidly in luminosity, an optimisation is required to perform a rapid and efficient followup of the skymap. Recent developments in both catalog of galaxies and galaxy targeting strategy already optimised significantly the follow-up of such event. Our work provides an efficient tool to upgrade in one hand a catalog of galaxies by adding the stellar mass information and on the other hand the galaxy targeting approach with a new expression of the grade using this stellar mass information to select and rank the galaxies. We crossmatched the GLADE and AllWISE catalogs to retrieve the WISE1 band luminosity to determine the stellar mass of $\sim 87 \%$ of the galaxies inside the GLADE catalog up to $400 \mathrm{Mpc}$. This catalog is complete in terms of stellar mass up to $\sim 33 \mathrm{Mpc}$, and up to $200 \mathrm{Mpc}$ if we consider galaxies contributing to half of the stellar mass density. The new formulation of the grade presented in this work allows not only to use the 3D position of galaxies to select them but also their stellar mass. We tested and validated our grade on the GW170817 event by showing an improvement on the ranking of interesting galaxies, i.e. massive galaxies, where NGC4993 is ranked first. This work plainly encourages further developments of the galaxy targeting strategy including other physical properties of the galaxies, for instance by focusing low SFR galaxies, but such development are slowed down by the poor number of information available in the publicly available galaxy catalogs. The Mangrove catalog is publicly available at https://mangrove.lal.in2p3.fr ${ }^{1}$, and this dedicated website automatically generates the list of galaxies, ranked by our new grade, compatible for each BNS event below 400Mpc, and observable from a given location on Earth. The improved grade presented in this work is implemented in the widely used gwemopt ${ }^{2}$ python package (Coughlin et al. 2018), developed to optimize the efforts of electromagnetic follow-up of gravitational wave events.

\section{ACKNOWLEDGEMENTS}

We acknowledge the Virtual data of labex P2IO for the supply of IT resources. This work made use of the NASA/IPAC Extragalactic Database (NED). We acknowledge the GRANDMA (Global Rapid Advanced Network Devoted to the Multi-messenger Addicts) collaboration for its technical support and assistance in the development of the mangrove web interface.

\section{REFERENCES}

Abbott B. P., et al., 2017a, Phys. Rev. Lett., 119, 161101

Abbott B. P., et al., 2017b, Nature, 551, 85

Abbott B. P., et al., 2017c, ApJ, 848, L13

Abbott B. P., et al., 2018a, Living Reviews in Relativity, 21, 3

1 The full catalog can be downloaded at https://mangrove.lal. in2p3.fr/download_full.php

2 https://github.com/mcoughlin/gwemopt 
Abbott B. P., et al., 2018b, Living Reviews in Relativity, 21, 3

Abbott B. P., et al., 2018c, Phys. Rev. Lett., 121, 161101

Antolini E., Caiazzo I., Davé R., Heyl J. S., 2017, MNRAS, 466, 2212

Arcavi I., et al., 2017, ApJ, 848, L33

Artale M. C., Mapelli M., Giacobbo N., Sabha N. B., Spera M., Santoliquido F., Bressan A., 2019, MNRAS, 487, 1675

Assef R. J., Stern D., Noirot G., Jun H. D., Cutri R. M., Eisenhardt P. R. M., 2018, ApJS, 234, 23

Berger E., 2014, ARA\&A, 52, 43

Blanchard P. K., et al., 2017, ApJ, 848, L22

Bruzual G., Charlot S., 2003, MNRAS, 344, 1000

Burtscher L., et al., 2015, A\&A, 578, A47

Calzetti D., Armus L., Bohlin R. C., Kinney A. L., Koornneef J., Storchi-Bergmann T., 2000, ApJ, 533, 682

Cardelli J. A., Clayton G. C., Mathis J. S., 1989, ApJ, 345, 245

Chen H.-Y., Holz D. E., Miller J., Evans M., Vitale S., Creighton J., 2017, arXiv e-prints, p. arXiv:1709.08079

Cook D. O., et al., 2019, ApJ, 880, 7

Coughlin M. W., et al., 2018, MNRAS, 478, 692

Coughlin M. W., Dietrich T., Heinzel J., Khetan N., Antier S., Christensen N., Coulter D. A., Foley R. J., 2019a, arXiv eprints, p. arXiv:1908.00889

Coughlin M. W., et al., 2019b, arXiv e-prints, p. arXiv:1909.01244

Cutri R. M., et al. 2014, VizieR Online Data Catalog, p. II/328

Dálya G., et al., 2018, MNRAS, 479, 2374

Fitzpatrick E. L., Massa D., 2007, ApJ, 663, 320

Fong W., et al., 2013, ApJ, 769, 56

Gehrels N., Cannizzo J. K., Kanner J., Kasliwal M. M., Nissanke S., Singer L. P., 2016, ApJ, 820, 136

Ghosh S., Bloemen S., Nelemans G., Groot P. J., Price L. R., 2016a, A\&A, 592, A82

Ghosh S., Bloemen S., Nelemans G., Groot P. J., Price L. R., 2016b, A\&A, 592, A82

Gill R., Nathanail A., Rezzolla L., 2019, ApJ, 876, 139

Górski K. M., Hivon E., Banday A. J., Wand elt B. D., Hansen F. K., Reinecke M., Bartelmann M., 2005, ApJ, 622, 759

Hajela A., et al., 2019, arXiv e-prints, p. arXiv:1909.06393

Hjorth J., et al., 2017, ApJ, 848, L31

Hotokezaka K., Nakar E., Gottlieb O., Nissanke S., Masuda K., Hallinan G., Mooley K. P., Deller A. T., 2019, Nature Astronomy, 3, 940

Ilbert O., et al., 2009, ApJ, 690, 1236

Im M., et al., 2017, ApJ, 849, L16

Jarrett T. H., et al., 2013, AJ, 145, 6

Kettlety T., et al., 2018, MNRAS, 473, 776

Leibler C. N., Berger E., 2010, ApJ, 725, 1202

Levan A. J., et al., 2017, ApJ, 848, L28

Mapelli M., Giacobbo N., Toffano M., Ripamonti E., Bressan A., Spera M., Branchesi M., 2018, MNRAS, 481, 5324

Maraston C., 2005, MNRAS, 362, 799

Metzger B. D., 2019, arXiv e-prints, p. arXiv:1910.01617

Norris M. A., Meidt S., Van de Ven G., Schinnerer E., Groves B., Querejeta M., 2014, ApJ, 797, 55

Pan Y. C., et al., 2017, ApJ, 848, L30

Planck Collaboration 2016, A\&A, 594, A13

Rana J., Mooley K. P., 2019, arXiv e-prints, p. arXiv:1904.07335

Ruiz A., Risaliti G., Nardini E., Panessa F., Carrera F. J., 2013, A\&A, 549, A125

Salafia O. S., Colpi M., Branchesi M., Chassande-Mottin E., Ghirlanda G., Ghisellini G., Vergani S. D., 2017, ApJ, 846, 62

Sanders D. B., 1999, in Terzian Y., Khachikian E., Weedman D., eds, IAU Symposium Vol. 194, Activity in Galaxies and Related Phenomena. p. 25 (arXiv:astro-ph/9903445)

Schechter P., 1976, ApJ, 203, 297

Singer L. P., Price L. R., 2016, Phys. Rev. D, 93, 024013

Singer L. P., et al., 2016, ApJ, 829, L15
Stern D., et al., 2012, ApJ, 753, 30

Toffano M., Mapelli M., Giacobbo N., Artale M. C., Ghirlanda G., 2019, MNRAS, p. 2085

Troja E., et al., 2018, Nature Communications, 9, 4089

Veitch J., et al., 2015, Phys. Rev. D, 91, 042003

Wright A. H., et al., 2017, MNRAS, 470, 283 
APPENDIX A:

Table A1: Ranking of the galaxies compatible with the GW170817 skymap according to grades defined in equations (1), (3) and (4), and the grade of (Arcavi et al. 2017).

\begin{tabular}{|c|c|c|c|c|c|c|c|c|c|c|}
\hline \multirow{2}{*}{ Galaxy name } & \multicolumn{2}{|c|}{$(1)$} & \multicolumn{2}{|c|}{ (Arcavi et al. 2017) } & \multicolumn{2}{|c|}{$(3)$} & \multicolumn{2}{|c|}{$(4)$} & \multirow{2}{*}{$\operatorname{BLum}\left(L_{\odot}\right)$} & \multirow{2}{*}{ Stellar mass $\left(M_{\odot}\right)$} \\
\hline & Rank & $P_{\text {loc }}$ & Rank & $G_{t o t}$ & Rank & $G_{t o t}$ & Rank & $G_{t o t}$ & & \\
\hline ESO575-053 & 1 & 0.06 & 8 & 0.043 & 6 & 0.038 & 6 & 0.05 & $8.32 \mathrm{e}+35$ & 9.675 \\
\hline PGC803966 & 2 & 0.059 & 35 & 0.003 & 35 & 0.001 & 8 & 0.033 & $5.96 \mathrm{e}+34$ & 7.835 \\
\hline WINGSJ125701.38-172325.2 & 3 & 0.059 & 59 & $<0.001$ & - & - & 9 & 0.033 & $1.52 \mathrm{e}+33$ & - \\
\hline ESO508-014 & 4 & 0.047 & 20 & 0.013 & 20 & 0.003 & 11 & 0.027 & $3.20 \mathrm{e}+35$ & 8.605 \\
\hline NGC4993 & 5 & 0.046 & 2 & 0.111 & 1 & 0.22 & 1 & 0.123 & $2.79 \mathrm{e}+36$ & 10.551 \\
\hline PGC797164 & 6 & 0.046 & 18 & 0.014 & 17 & 0.005 & 10 & 0.028 & $3.60 \mathrm{e}+35$ & 8.864 \\
\hline ESO508-004 & 7 & 0.045 & 14 & 0.016 & 26 & 0.002 & 12 & 0.026 & $4.17 \mathrm{e}+35$ & 8.416 \\
\hline IC4197 & 8 & 0.04 & 1 & 0.137 & 2 & 0.195 & 2 & 0.109 & $3.96 \mathrm{e}+36$ & 10.563 \\
\hline ESO508-019 & 9 & 0.04 & 5 & 0.064 & 16 & 0.005 & 13 & 0.024 & $1.87 \mathrm{e}+36$ & 9.004 \\
\hline 2MASS 13104593-2351566 & 10 & 0.038 & 13 & 0.027 & - & - & 14 & 0.021 & $8.14 \mathrm{e}+35$ & - \\
\hline 796755 & 11 & 0.037 & 44 & 0.001 & 38 & $<0.001$ & 15 & 0.02 & $3.43 \mathrm{e}+34$ & 7.9711 \\
\hline NGC4968 & 12 & 0.036 & 4 & 0.072 & - & - & 16 & 0.02 & $2.29 \mathrm{e}+36$ & - \\
\hline 6dFJ1309178-242256 & 13 & 0.034 & 33 & 0.003 & 36 & 0.001 & 17 & 0.019 & $1.05 \mathrm{e}+35$ & 8.075 \\
\hline ESO508-010 & 14 & 0.033 & 11 & 0.04 & 7 & 0.036 & 7 & 0.035 & $1.39 \mathrm{e}+36$ & 9.914 \\
\hline PGC169663 & 15 & 0.031 & 42 & 0.001 & 30 & 0.001 & 18 & 0.018 & $4.26 \mathrm{e}+34$ & 8.171 \\
\hline IC4180 & 16 & 0.027 & 6 & 0.063 & 5 & 0.105 & 4 & 0.062 & $2.73 \mathrm{e}+36$ & 10.466 \\
\hline PGC043966 & 17 & 0.024 & 15 & 0.016 & 29 & 0.001 & 20 & 0.014 & $7.77 \mathrm{e}+35$ & 8.574 \\
\hline PGC799951 & 18 & 0.021 & 36 & 0.003 & 37 & $<0.001$ & 22 & 0.012 & $1.66 \mathrm{e}+35$ & 8.247 \\
\hline WINGSJ125701.40-172325.3 & 19 & 0.021 & - & - & - & - & 23 & 0.011 & - & - \\
\hline ESO508-015 & 20 & 0.02 & 19 & 0.013 & 42 & $<0.001$ & 24 & 0.011 & $8.01 \mathrm{e}+35$ & 7.885 \\
\hline ESO508-024 & 21 & 0.019 & 10 & 0.04 & - & - & 26 & 0.011 & $2.45 \mathrm{e}+36$ & - \\
\hline ESO575-029 & 22 & 0.019 & 9 & 0.042 & 15 & 0.008 & 19 & 0.014 & $2.59 \mathrm{e}+36$ & 9.49 \\
\hline PGC169670 & 23 & 0.017 & 40 & 0.002 & 31 & 0.001 & 28 & 0.01 & $1.05 \mathrm{e}+35$ & 8.428 \\
\hline PGC772879 & 24 & 0.017 & 46 & 0.001 & 46 & $<0.001$ & 29 & 0.01 & $5.72 \mathrm{e}+34$ & 7.862 \\
\hline NGC4970 & 25 & 0.017 & 3 & 0.081 & 3 & 0.139 & 3 & 0.071 & $5.54 \mathrm{e}+36$ & 10.791 \\
\hline WINGSJ125701.40-172325.3 & 26 & 0.015 & - & - & - & - & 31 & 0.008 & - & - \\
\hline NGC4830 & 27 & 0.011 & 7 & 0.048 & 4 & 0.11 & 5 & 0.055 & $5.16 \mathrm{e}+36$ & 10.882 \\
\hline PGC043664 & 28 & 0.01 & 16 & 0.015 & 14 & 0.01 & 27 & 0.01 & $1.71 \mathrm{e}+36$ & 9.874 \\
\hline ESO575-061 & 29 & 0.01 & 37 & 0.002 & 43 & $<0.001$ & 34 & 0.006 & $2.74 \mathrm{e}+35$ & 8.156 \\
\hline PGC044023 & 30 & 0.009 & 43 & 0.001 & 32 & 0.001 & 36 & 0.005 & $1.41 \mathrm{e}+35$ & 8.693 \\
\hline PGC044312 & 31 & 0.008 & 39 & 0.002 & 23 & 0.002 & 37 & 0.005 & $2.86 \mathrm{e}+35$ & 9.321 \\
\hline PGC044500 & 32 & 0.007 & 25 & 0.007 & 33 & 0.001 & 38 & 0.004 & $1.08 \mathrm{e}+36$ & 8.787 \\
\hline PGC044021 & 33 & 0.006 & 26 & 0.006 & 28 & 0.001 & 39 & 0.004 & $1.16 \mathrm{e}+36$ & 9.188 \\
\hline ESO508-033 & 34 & 0.006 & 24 & 0.007 & 12 & 0.012 & 30 & 0.009 & $1.29 \mathrm{e}+36$ & 10.152 \\
\hline WINGSJ125217.42-153054.2 & 35 & 0.006 & - & - & 40 & $<0.001$ & 40 & 0.003 & - & 8.431 \\
\hline ABELL_1644:[D80]141 & 36 & 0.005 & - & - & - & - & 42 & 0.003 & - & - \\
\hline ESO508-011 & 37 & 0.005 & 32 & 0.003 & 47 & $<0.001$ & 45 & 0.003 & $8.54 \mathrm{e}+35$ & 8.302 \\
\hline PGC044478 & 38 & 0.004 & 29 & 0.004 & 45 & $<0.001$ & 46 & 0.002 & $1.08 \mathrm{e}+36$ & 8.479 \\
\hline IC3799 & 39 & 0.004 & 12 & 0.028 & 11 & 0.012 & 33 & 0.008 & $8.50 \mathrm{e}+36$ & 10.37 \\
\hline PGC183552 & 40 & 0.004 & 38 & 0.002 & 24 & 0.002 & 43 & 0.003 & $7.35 \mathrm{e}+35$ & 9.568 \\
\hline ESO508-003 & 41 & 0.003 & 23 & 0.007 & 22 & 0.002 & 44 & 0.003 & $2.94 \mathrm{e}+36$ & 9.754 \\
\hline NGC4763 & 42 & 0.003 & 17 & 0.015 & 9 & 0.02 & 25 & 0.011 & $6.15 \mathrm{e}+36$ & 10.729 \\
\hline PGC044234 & 43 & 0.003 & 27 & 0.005 & 25 & 0.002 & 47 & 0.002 & $2.12 \mathrm{e}+36$ & 9.679 \\
\hline ESO508-007 & 44 & 0.003 & 49 & 0.001 & 53 & $<0.001$ & 50 & 0.001 & $2.82 \mathrm{e}+35$ & 7.609 \\
\hline PGC043908 & 45 & 0.003 & 30 & 0.004 & 18 & 0.003 & 41 & 0.003 & $1.68 \mathrm{e}+36$ & 10.0 \\
\hline ESO575-035 & 46 & 0.002 & 34 & 0.003 & 39 & $<0.001$ & 51 & 0.001 & $1.66 \mathrm{e}+36$ & 9.018 \\
\hline PGC043424 & 47 & 0.002 & 22 & 0.008 & 8 & 0.027 & 21 & 0.013 & $5.32 \mathrm{e}+36$ & 11.051 \\
\hline IC3831 & 48 & 0.002 & 28 & 0.004 & 13 & 0.011 & 35 & 0.006 & $3.07 \mathrm{e}+36$ & 10.698 \\
\hline PGC043505 & 49 & 0.002 & 55 & $<0.001$ & 44 & $<0.001$ & 53 & 0.001 & $1.66 \mathrm{e}+35$ & 8.964 \\
\hline NGC4756 & 50 & 0.001 & 21 & 0.009 & 10 & 0.016 & 32 & 0.008 & $6.89 \mathrm{e}+36$ & 10.904 \\
\hline PGC043344 & 51 & 0.001 & 47 & 0.001 & - & - & 54 & 0.001 & $5.20 \mathrm{e}+35$ & - \\
\hline WINGSJ125252.62-152426.5 & 52 & 0.001 & - & - & - & - & 55 & 0.001 & - & - \\
\hline ESO508-020 & 53 & 0.001 & 48 & 0.001 & - & - & 58 & 0.001 & $5.69 \mathrm{e}+35$ & - \\
\hline PGC910856 & 54 & 0.001 & 57 & $<0.001$ & 49 & $<0.001$ & 56 & 0.001 & $1.06 \mathrm{e}+35$ & 8.75 \\
\hline PGC908166 & 55 & 0.001 & 56 & $<0.001$ & 48 & $<0.001$ & 57 & 0.001 & $1.71 \mathrm{e}+35$ & 8.763 \\
\hline PGC043823 & 56 & 0.001 & 45 & 0.001 & 27 & 0.001 & 52 & 0.001 & $1.03 \mathrm{e}+36$ & 9.937 \\
\hline PGC046026 & 57 & 0.001 & 41 & 0.001 & 21 & 0.002 & 49 & 0.002 & $1.72 \mathrm{e}+36$ & 10.22 \\
\hline NGC4724 & 58 & 0.001 & 31 & 0.004 & 19 & 0.003 & 48 & 0.002 & $4.58 \mathrm{e}+36$ & 10.365 \\
\hline PGC170205 & 59 & 0.001 & 50 & 0.001 & - & - & 60 & $<0.001$ & $1.01 \mathrm{e}+36$ & - \\
\hline
\end{tabular}




\begin{tabular}{|c|c|c|c|c|c|c|c|c|c|c|}
\hline \multirow{2}{*}{ Galaxy name } & \multicolumn{2}{|c|}{ (1) } & \multicolumn{2}{|c|}{ (Arcavi et al. 2017) } & \multicolumn{2}{|c|}{$(3)$} & \multicolumn{2}{|c|}{ (4) } & \multirow{2}{*}{$\operatorname{BLum}\left(L_{\odot}\right)$} & \multirow{2}{*}{ Stellar mass $\left(\boldsymbol{M}_{\odot}\right)$} \\
\hline & Rank & $P_{\text {loc }}$ & Rank & $G_{\text {tot }}$ & Rank & $G_{t o t}$ & Rank & $G_{\text {tot }}$ & & \\
\hline PGC043913 & 60 & 0.001 & 51 & $<0.001$ & 34 & 0.001 & 59 & 0.001 & $9.78 \mathrm{e}+35$ & 9.87 \\
\hline PGC937614 & 61 & $<0.001$ & 53 & $<0.001$ & 41 & $<0.001$ & 61 & $<0.001$ & $1.36 \mathrm{e}+36$ & 9.79 \\
\hline PGC943386 & 62 & $<0.001$ & 58 & $<0.001$ & 51 & $<0.001$ & 62 & $<0.001$ & $4.77 \mathrm{e}+35$ & 8.724 \\
\hline ESO575-041 & 63 & $<0.001$ & 54 & $<0.001$ & 50 & $<0.001$ & 63 & $<0.001$ & $1.20 \mathrm{e}+36$ & 8.879 \\
\hline 2MASS 12492243-1321162 & 64 & $<0.001$ & 52 & $<0.001$ & - & - & 64 & $<0.001$ & $2.01 \mathrm{e}+36$ & - \\
\hline PGC942354 & 65 & $<0.001$ & 60 & $<0.001$ & 52 & $<0.001$ & 65 & $<0.001$ & $3.23 \mathrm{e}+35$ & 8.723 \\
\hline
\end{tabular}

\section{APPENDIX B:}

Table B1: Ranking of the galaxies compatible with the GW170817 without virgo data skymap according to grades defined in equations (1), (3) and (4), and the grade of (Arcavi et al. 2017).






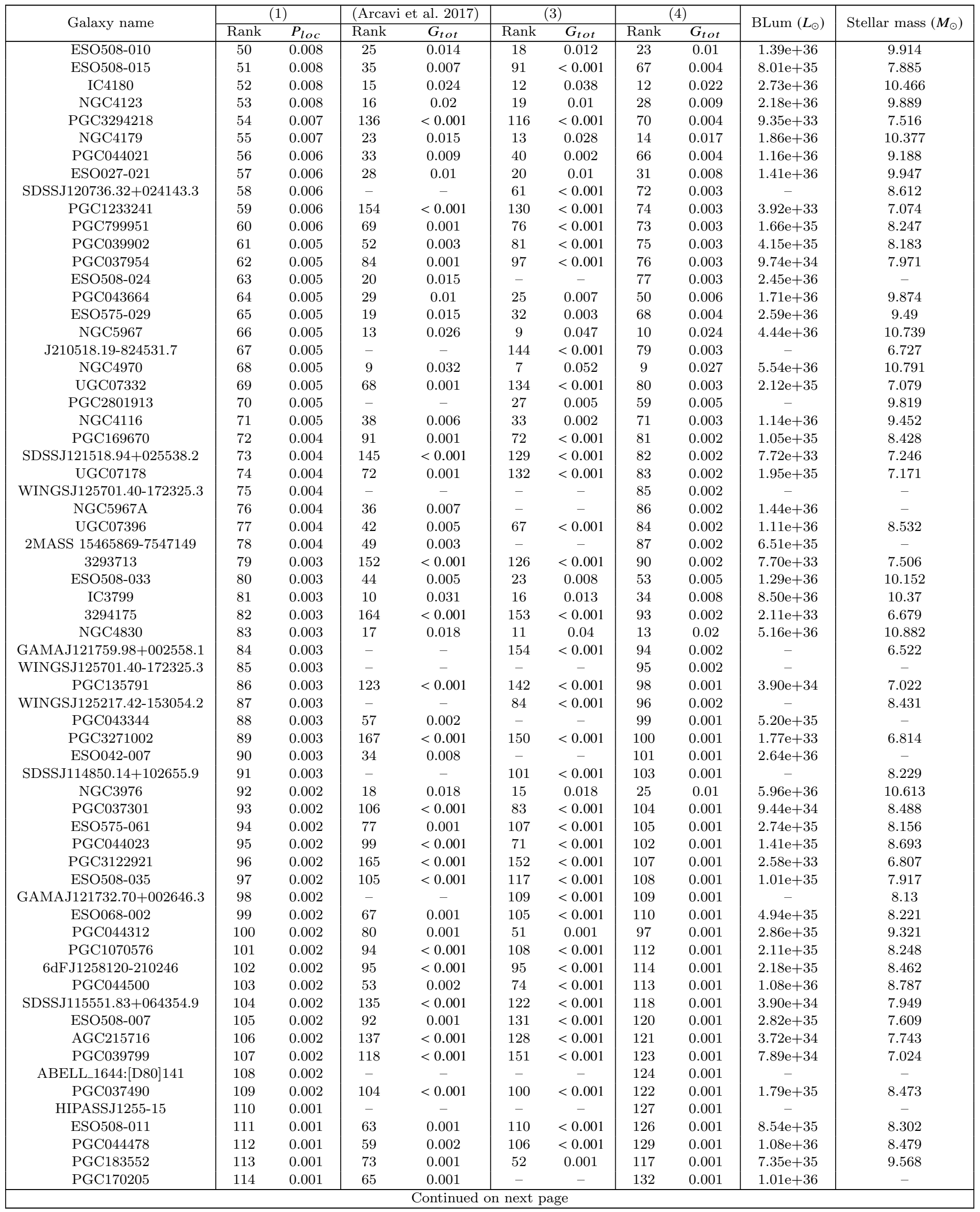




\begin{tabular}{|c|c|c|c|c|c|c|c|c|c|c|}
\hline \multirow{2}{*}{ Galaxy name } & \multicolumn{2}{|c|}{ (1) } & \multicolumn{2}{|c|}{ (Arcavi et al. 2017) } & \multicolumn{2}{|c|}{ (3) } & \multicolumn{2}{|c|}{ (4) } & \multirow{2}{*}{ BLum $\left(L_{\odot}\right)$} & \multirow{2}{*}{ Stellar mass $\left(M_{\odot}\right)$} \\
\hline & Rank & $P_{\text {loc }}$ & Rank & $G_{t o t}$ & Rank & $G_{\text {tot }}$ & Rank & $G_{\text {tot }}$ & & \\
\hline IC3831 & 115 & 0.001 & 46 & 0.004 & 21 & 0.009 & 58 & 0.005 & $3.07 \mathrm{e}+36$ & 10.698 \\
\hline PGC043424 & 116 & 0.001 & 37 & 0.007 & 14 & 0.02 & 22 & 0.01 & $5.32 \mathrm{e}+36$ & 11.051 \\
\hline PGC1031551 & 117 & 0.001 & 163 & $<0.001$ & 159 & $<0.001$ & 134 & $<0.001$ & $7.36 \mathrm{e}+33$ & 6.862 \\
\hline PGC3294523 & 118 & 0.001 & 172 & $<0.001$ & 164 & $<0.001$ & 136 & $<0.001$ & $1.86 \mathrm{e}+33$ & 6.498 \\
\hline ESO508-003 & 119 & 0.001 & 51 & 0.003 & 50 & 0.001 & 125 & 0.001 & $2.94 \mathrm{e}+36$ & 9.754 \\
\hline PGC720745 & 120 & 0.001 & 153 & $<0.001$ & 140 & $<0.001$ & 138 & $<0.001$ & $3.11 \mathrm{e}+34$ & 7.62 \\
\hline IC0874 & 121 & 0.001 & 75 & 0.001 & 43 & 0.001 & 111 & 0.001 & $8.89 \mathrm{e}+35$ & 10.016 \\
\hline PGC758254 & 122 & 0.001 & 156 & $<0.001$ & 146 & $<0.001$ & 139 & $<0.001$ & $2.69 \mathrm{e}+34$ & 7.49 \\
\hline NGC4763 & 123 & 0.001 & 41 & 0.006 & 24 & 0.007 & 69 & 0.004 & $6.15 \mathrm{e}+36$ & 10.729 \\
\hline PGC043908 & 124 & 0.001 & 60 & 0.001 & 45 & 0.001 & 116 & 0.001 & $1.68 \mathrm{e}+36$ & 10.0 \\
\hline PGC3293619 & 125 & 0.001 & 180 & $<0.001$ & 162 & $<0.001$ & 141 & $<0.001$ & $6.67 \mathrm{e}+32$ & 6.631 \\
\hline PGC046026 & 126 & 0.001 & 64 & 0.001 & 36 & 0.002 & 106 & 0.001 & $1.72 \mathrm{e}+36$ & 10.22 \\
\hline NGC4724 & 127 & 0.001 & 48 & 0.004 & 31 & 0.003 & 92 & 0.002 & $4.58 \mathrm{e}+36$ & 10.365 \\
\hline PGC685308 & 128 & 0.001 & 130 & $<0.001$ & 104 & $<0.001$ & 142 & $<0.001$ & $1.21 \mathrm{e}+35$ & 8.765 \\
\hline PGC044234 & 129 & 0.001 & 58 & 0.002 & 57 & 0.001 & 131 & 0.001 & $2.12 \mathrm{e}+36$ & 9.679 \\
\hline PGC091191 & 130 & 0.001 & 168 & $<0.001$ & 163 & $<0.001$ & 145 & $<0.001$ & $5.86 \mathrm{e}+33$ & 6.666 \\
\hline NGC4756 & 131 & 0.001 & 43 & 0.005 & 22 & 0.008 & 65 & 0.004 & $6.89 \mathrm{e}+36$ & 10.904 \\
\hline WINGSJ125252.62-152426.5 & 132 & 0.001 & - & - & - & - & 148 & $<0.001$ & - & - \\
\hline PGC3291384 & 133 & 0.001 & 179 & $<0.001$ & 170 & $<0.001$ & 149 & $<0.001$ & $8.83 \mathrm{e}+32$ & 6.295 \\
\hline 135794 & 134 & 0.001 & 174 & $<0.001$ & 166 & $<0.001$ & 150 & $<0.001$ & $2.31 \mathrm{e}+33$ & 6.473 \\
\hline 2MASXJ13242754-3025548 & 135 & 0.001 & 128 & $<0.001$ & - & - & 151 & $<0.001$ & $1.50 \mathrm{e}+35$ & - \\
\hline PGC043505 & 136 & 0.001 & 125 & $<0.001$ & 98 & $<0.001$ & 147 & $<0.001$ & $1.66 \mathrm{e}+35$ & 8.964 \\
\hline ESO575-035 & 137 & 0.001 & 70 & 0.001 & 94 & $<0.001$ & 146 & $<0.001$ & $1.66 \mathrm{e}+36$ & 9.018 \\
\hline PGC043823 & 138 & 0.001 & 81 & 0.001 & 48 & 0.001 & 130 & 0.001 & $1.03 \mathrm{e}+36$ & 9.937 \\
\hline NGC4504 & 139 & 0.001 & 86 & 0.001 & 68 & $<0.001$ & 140 & $<0.001$ & $9.58 \mathrm{e}+35$ & 9.402 \\
\hline PGC3294233 & 140 & 0.001 & 178 & $<0.001$ & - & - & 152 & $<0.001$ & $9.60 \mathrm{e}+32$ & - \\
\hline 2MASXJ12490814-1124354 & 141 & 0.001 & 131 & $<0.001$ & - & - & 154 & $<0.001$ & $1.50 \mathrm{e}+35$ & - \\
\hline PGC104686 & 142 & 0.001 & 148 & $<0.001$ & - & - & 155 & $<0.001$ & $5.98 \mathrm{e}+34$ & - \\
\hline NGC5114 & 143 & 0.001 & 50 & 0.003 & 26 & 0.005 & 78 & 0.003 & $4.65 \mathrm{e}+36$ & 10.774 \\
\hline ESO508-036 & 144 & $<0.001$ & 146 & $<0.001$ & 138 & $<0.001$ & 156 & $<0.001$ & $6.54 \mathrm{e}+34$ & 7.924 \\
\hline PGC910856 & 145 & $<0.001$ & 139 & $<0.001$ & 111 & $<0.001$ & 153 & $<0.001$ & $1.06 \mathrm{e}+35$ & 8.75 \\
\hline PGC041725 & 146 & $<0.001$ & 133 & $<0.001$ & 157 & $<0.001$ & 158 & $<0.001$ & $1.60 \mathrm{e}+35$ & 7.166 \\
\hline NGC4487 & 147 & $<0.001$ & 85 & 0.001 & 65 & $<0.001$ & 144 & $<0.001$ & $1.14 \mathrm{e}+36$ & 9.493 \\
\hline PGC908166 & 148 & $<0.001$ & 134 & $<0.001$ & 113 & $<0.001$ & 159 & $<0.001$ & $1.71 \mathrm{e}+35$ & 8.763 \\
\hline PGC043913 & 149 & $<0.001$ & 96 & $<0.001$ & 58 & 0.001 & 135 & $<0.001$ & $9.78 \mathrm{e}+35$ & 9.87 \\
\hline PGC3097711 & 150 & $<0.001$ & 181 & $<0.001$ & 173 & $<0.001$ & 161 & $<0.001$ & $1.14 \mathrm{e}+33$ & 6.106 \\
\hline PGC141593 & 151 & $<0.001$ & 126 & $<0.001$ & 112 & $<0.001$ & 160 & $<0.001$ & $2.23 \mathrm{e}+35$ & 8.8 \\
\hline PGC3097710 & 152 & $<0.001$ & 182 & $<0.001$ & - & - & 164 & $<0.001$ & $1.14 \mathrm{e}+33$ & - \\
\hline PGC705472 & 153 & $<0.001$ & 144 & $<0.001$ & 156 & $<0.001$ & 163 & $<0.001$ & $9.78 \mathrm{e}+34$ & 7.363 \\
\hline PGC937614 & 154 & $<0.001$ & 90 & 0.001 & 62 & $<0.001$ & 143 & $<0.001$ & $1.36 \mathrm{e}+36$ & 9.79 \\
\hline ESO508-020 & 155 & $<0.001$ & 110 & $<0.001$ & - & - & 165 & $<0.001$ & $5.69 \mathrm{e}+35$ & - \\
\hline NGC5061 & 156 & $<0.001$ & 54 & 0.002 & 29 & 0.004 & 89 & 0.002 & $4.95 \mathrm{e}+36$ & 10.805 \\
\hline PGC135798 & 157 & $<0.001$ & 171 & $<0.001$ & 167 & $<0.001$ & 168 & $<0.001$ & $4.70 \mathrm{e}+33$ & 6.665 \\
\hline PGC042120 & 158 & $<0.001$ & 173 & $<0.001$ & 169 & $<0.001$ & 169 & $<0.001$ & $3.98 \mathrm{e}+33$ & 6.529 \\
\hline ESO444-026 & 159 & $<0.001$ & 56 & 0.002 & - & - & 170 & $<0.001$ & $4.64 \mathrm{e}+36$ & - \\
\hline J132249.66-300651.8 & 160 & $<0.001$ & - & - & 119 & $<0.001$ & 166 & $<0.001$ & - & 8.706 \\
\hline PGC943386 & 161 & $<0.001$ & 114 & $<0.001$ & 118 & $<0.001$ & 167 & $<0.001$ & $4.77 \mathrm{e}+35$ & 8.724 \\
\hline PGC3097712 & 162 & $<0.001$ & 177 & $<0.001$ & 174 & $<0.001$ & 172 & $<0.001$ & $1.80 \mathrm{e}+33$ & 6.13 \\
\hline PGC3294387 & 163 & $<0.001$ & 184 & $<0.001$ & 172 & $<0.001$ & 171 & $<0.001$ & $4.59 \mathrm{e}+32$ & 6.383 \\
\hline PGC3268622 & 164 & $<0.001$ & 183 & $<0.001$ & 160 & $<0.001$ & 173 & $<0.001$ & $5.02 \mathrm{e}+32$ & 7.236 \\
\hline PGC3097709 & 165 & $<0.001$ & 176 & $<0.001$ & 171 & $<0.001$ & 174 & $<0.001$ & $2.38 \mathrm{e}+33$ & 6.406 \\
\hline PGC046803 & 166 & $<0.001$ & 93 & $<0.001$ & 53 & 0.001 & 133 & $<0.001$ & $1.43 \mathrm{e}+36$ & 10.154 \\
\hline PGC104868 & 167 & $<0.001$ & 158 & $<0.001$ & 148 & $<0.001$ & 175 & $<0.001$ & $4.53 \mathrm{e}+34$ & 7.848 \\
\hline ESO444-021 & 168 & $<0.001$ & 66 & 0.001 & 87 & $<0.001$ & 162 & $<0.001$ & $4.08 \mathrm{e}+36$ & 9.381 \\
\hline WINGSJ132507.84-315046.2 & 169 & $<0.001$ & - & - & - & - & 177 & $<0.001$ & - & - \\
\hline NGC5078 & 170 & $<0.001$ & 55 & 0.002 & 28 & 0.004 & 88 & 0.002 & $6.52 \mathrm{e}+36$ & 10.978 \\
\hline ESO444-011 & 171 & $<0.001$ & 111 & $<0.001$ & 120 & $<0.001$ & 176 & $<0.001$ & $7.74 \mathrm{e}+35$ & 8.824 \\
\hline ESO508-039 & 172 & $<0.001$ & 151 & $<0.001$ & 158 & $<0.001$ & 179 & $<0.001$ & $1.09 \mathrm{e}+35$ & 7.434 \\
\hline NGC4748 & 173 & $<0.001$ & 82 & 0.001 & 37 & 0.002 & 115 & 0.001 & $2.32 \mathrm{e}+36$ & 10.672 \\
\hline J132044.59-302043.7 & 174 & $<0.001$ & - & - & - & - & 181 & $<0.001$ & - & - \\
\hline ESO444-012 & 175 & $<0.001$ & 78 & 0.001 & 56 & 0.001 & 137 & $<0.001$ & $3.17 \mathrm{e}+36$ & 10.26 \\
\hline PGC141595 & 176 & $<0.001$ & 141 & $<0.001$ & 121 & $<0.001$ & 180 & $<0.001$ & $2.06 \mathrm{e}+35$ & 8.881 \\
\hline PGC850539 & 177 & $<0.001$ & 122 & $<0.001$ & 127 & $<0.001$ & 183 & $<0.001$ & $5.31 \mathrm{e}+35$ & 8.668 \\
\hline PGC740755 & 178 & $<0.001$ & 132 & $<0.001$ & - & - & 184 & $<0.001$ & $3.76 \mathrm{e}+35$ & - \\
\hline ESO444-002 & 179 & $<0.001$ & 157 & $<0.001$ & 161 & $<0.001$ & 186 & $<0.001$ & $1.03 \mathrm{e}+35$ & 7.375 \\
\hline & & & & 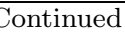 & nex & & & & & \\
\hline
\end{tabular}




\begin{tabular}{|c|c|c|c|c|c|c|c|c|c|c|}
\hline \multirow{2}{*}{ Galaxy name } & \multicolumn{2}{|c|}{ (1) } & \multicolumn{2}{|c|}{ (Arcavi et al. 2017) } & \multicolumn{2}{|c|}{$(3)$} & \multicolumn{2}{|c|}{ (4) } & \multirow{2}{*}{$\operatorname{BLum}\left(L_{\odot}\right)$} & \multirow{2}{*}{ Stellar mass $\left(M_{\odot}\right)$} \\
\hline & Rank & $P_{\text {loc }}$ & Rank & $G_{t o t}$ & Rank & $G_{t o t}$ & Rank & $G_{t o t}$ & & \\
\hline PGC763675 & 180 & $<0.001$ & 159 & $<0.001$ & 155 & $<0.001$ & 187 & $<0.001$ & $6.22 \mathrm{e}+34$ & 7.643 \\
\hline NGC5124 & 181 & $<0.001$ & 62 & 0.001 & 30 & 0.003 & 91 & 0.002 & $6.38 \mathrm{e}+36$ & 11.025 \\
\hline ESO444-033 & 182 & $<0.001$ & 140 & $<0.001$ & 147 & $<0.001$ & 188 & $<0.001$ & $2.69 \mathrm{e}+35$ & 8.095 \\
\hline WINGSJ132507.84-315046.2 & 183 & $<0.001$ & - & - & - & - & 189 & $<0.001$ & - & - \\
\hline WINGSJ132507.85-315046.2 & 184 & $<0.001$ & 175 & $<0.001$ & - & - & 190 & $<0.001$ & $6.71 \mathrm{e}+33$ & - \\
\hline PGC141602 & 185 & $<0.001$ & 138 & $<0.001$ & 143 & $<0.001$ & 191 & $<0.001$ & $3.89 \mathrm{e}+35$ & 8.239 \\
\hline NGC5048 & 186 & $<0.001$ & 74 & 0.001 & 44 & 0.001 & 128 & 0.001 & $5.21 \mathrm{e}+36$ & 10.713 \\
\hline PGC732248 & 187 & $<0.001$ & 143 & $<0.001$ & 139 & $<0.001$ & 192 & $<0.001$ & $2.49 \mathrm{e}+35$ & 8.403 \\
\hline SDSSJ120133.99+042759.3 & 188 & $<0.001$ & 160 & $<0.001$ & 137 & $<0.001$ & 194 & $<0.001$ & $7.38 \mathrm{e}+34$ & 8.456 \\
\hline NGC5051 & 189 & $<0.001$ & 76 & 0.001 & 39 & 0.002 & 119 & 0.001 & $4.78 \mathrm{e}+36$ & 10.85 \\
\hline PGC046903 & 190 & $<0.001$ & 150 & $<0.001$ & - & - & 195 & $<0.001$ & $1.95 \mathrm{e}+35$ & - \\
\hline IC0879 & 191 & $<0.001$ & 147 & $<0.001$ & 115 & $<0.001$ & 193 & $<0.001$ & $2.54 \mathrm{e}+35$ & 9.272 \\
\hline PGC141596 & 192 & $<0.001$ & 121 & $<0.001$ & 85 & $<0.001$ & 182 & $<0.001$ & $9.42 \mathrm{e}+35$ & 9.764 \\
\hline PGC722221 & 193 & $<0.001$ & 170 & $<0.001$ & 168 & $<0.001$ & 198 & $<0.001$ & $2.70 \mathrm{e}+34$ & 7.113 \\
\hline PGC117211 & 194 & $<0.001$ & 162 & $<0.001$ & 136 & $<0.001$ & 197 & $<0.001$ & $8.52 \mathrm{e}+34$ & 8.687 \\
\hline ESO444-015 & 195 & $<0.001$ & 107 & $<0.001$ & 75 & $<0.001$ & 178 & $<0.001$ & $2.42 \mathrm{e}+36$ & 10.053 \\
\hline HIPASSJ1457-67 & 196 & $<0.001$ & - & - & 133 & $<0.001$ & 196 & $<0.001$ & - & 8.823 \\
\hline PGC042964 & 197 & $<0.001$ & 166 & $<0.001$ & 165 & $<0.001$ & 199 & $<0.001$ & $6.01 \mathrm{e}+34$ & 7.317 \\
\hline ESO575-041 & 198 & $<0.001$ & 127 & $<0.001$ & 135 & $<0.001$ & 200 & $<0.001$ & $1.20 \mathrm{e}+36$ & 8.879 \\
\hline PGC939548 & 199 & $<0.001$ & 149 & $<0.001$ & 141 & $<0.001$ & 201 & $<0.001$ & $4.06 \mathrm{e}+35$ & 8.62 \\
\hline PGC1295846 & 200 & $<0.001$ & 161 & $<0.001$ & 145 & $<0.001$ & 203 & $<0.001$ & $1.76 \mathrm{e}+35$ & 8.642 \\
\hline PGC2793691 & 201 & $<0.001$ & 142 & $<0.001$ & - & - & 204 & $<0.001$ & $7.10 \mathrm{e}+35$ & - \\
\hline ESO443-086 & 202 & $<0.001$ & 124 & $<0.001$ & 124 & $<0.001$ & 202 & $<0.001$ & $1.88 \mathrm{e}+36$ & 9.436 \\
\hline PGC043964 & 203 & $<0.001$ & 120 & $<0.001$ & 77 & $<0.001$ & 185 & $<0.001$ & $2.56 \mathrm{e}+36$ & 10.361 \\
\hline NGC5126 & 204 & $<0.001$ & 109 & $<0.001$ & 60 & $<0.001$ & 157 & $<0.001$ & $4.67 \mathrm{e}+36$ & 10.816 \\
\hline PGC104887 & 205 & $<0.001$ & 169 & $<0.001$ & 149 & $<0.001$ & 205 & $<0.001$ & $8.69 \mathrm{e}+34$ & 8.616 \\
\hline
\end{tabular}

